Article

\title{
Regular Exercise and Weight-Control Behavior Are Protective Factors against Osteoporosis for General Population: A Propensity Score-Matched Analysis from Taiwan Biobank Participants
}

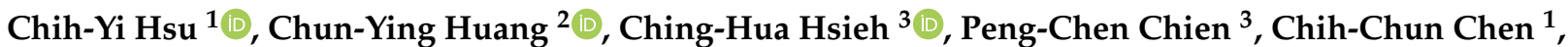 \\ Shao-Yun Hou ${ }^{1}$ and Shao-Chun $\mathrm{Wu}^{1, *(D)}$
}

Citation: Hsu, C.-Y.; Huang, C.-Y.; Hsieh, C.-H.; Chien, P.-C.; Chen, C.-C.; Hou, S.-Y.; Wu, S.-C. Regular Exercise and Weight-Control Behavior Are Protective Factors against Osteoporosis for General Population: A Propensity

Score-Matched Analysis from Taiwan Biobank Participants. Nutrients 2022, 14, 641. https://doi.org/10.3390/ nu14030641

Academic Editor: Wolfgang Kemmler

Received: 12 December 2021

Accepted: 27 January 2022

Published: 2 February 2022

Publisher's Note: MDPI stays neutral with regard to jurisdictional claims in published maps and institutional affiliations.

Copyright: (C) 2022 by the authors. Licensee MDPI, Basel, Switzerland. This article is an open access article distributed under the terms and conditions of the Creative Commons Attribution (CC BY) license (https:// creativecommons.org/licenses/by/ $4.0 /)$.
1 Department of Anesthesiology, Kaohsiung Chang Gung Memorial and Chang Gung University College of Medicine, No. 123, Ta-Pei Rd., Niao-Song Dist., Kaohsiung City 833401, Taiwan; hsuchiyi@cgmh.org.tw (C.-Y.H.); b9702062@cgmh.org.tw (C.-C.C.); walkings@cgmh.org.tw (S.-Y.H.)

2 Department of Trauma Surgery, Kaohsiung Chang Gung Memorial and Chang Gung University College of Medicine, No. 123, Ta-Pei Rd., Niao-Song Dist., Kaohsiung City 833401, Taiwan; junyinhaung@yahoo.com.tw

3 Department of Plastic Surgery, Kaohsiung Chang Gung Memorial and Chang Gung University College of Medicine, No. 123, Ta-Pei Rd., Niao-Song Dist., Kaohsiung City 833401, Taiwan; m93chinghua@gmail.com (C.-H.H.); VENU_CHIEN@hotmail.com (P.-C.C.)

* Correspondence: shaochunwu@gmail.com; Tel.: +886-7-7317123 (ext. 2788); Fax: +886-7-7351638

\begin{abstract}
The rising prevalence of osteoporosis, which can lead to osteoporotic fractures, increases morbidity, mortality, and socioeconomic burden. Multiple factors influencing bone mass have already been identified. The aim of this study was to investigate whether exercise habits and weight-control behaviors can lower the incidence of osteoporosis in the general population. This retrospective study recruited all participants aged 35-70 years who underwent dual-energy X-ray absorptiometry (DXA) from Taiwan Biobank (TWB). The final analysis consisted of 3320 eligible participants divided into two groups; demographic characteristics, prevalence of clinical symptoms, comorbidities, and daily behavior were collected using a self-reported questionnaire. After propensity score matching with a 1:1 ratio, 1107 out of 2214 individuals were classified into the osteoporosis group. Age, body fat rate, body shape, diabetes mellitus, and social status were found to affect the incidence of osteoporosis. Subjects with a habit of regular exercise and weight-control behavior showed decreased odds of osteoporosis. (odds ratio: 0.709 and $0.753,95 \%$ confidence interval: 0.599-0.839 and $0.636-0.890)$. In the general population, regular exercise or weight-control behavior lowers the incidence of osteoporosis.
\end{abstract}

Keywords: osteoporosis; osteoporotic fracture; exercise; weight control; Taiwan biobank; dual-energy X-ray absorptiometry

\section{Introduction}

Osteoporosis is a major public health problem, and a projected rising prevalence is expected in an aged society [1]. However, it is often asymptomatic until osteoporotic fractures occur [2]. According to statistics from the International Osteoporosis Foundation (IOF), 158 million adults aged 50 years and above are at high risk of osteoporotic fracture worldwide, and the number is predicted to double by 2040. Osteoporosis is prevalent in women and the elderly, but it also occurs in all populations and at all ages, and osteoporotic fractures cause significant morbidity, mortality and psychosocial and financial consequences [3]. In the UK, the National Health Service is estimated to spend GPB 4.4 billion per year because of fragility fractures [4]. In the USA, the cost of treatment for osteoporotic fractures is expected to reach USD 95 billion annually by 2040 [5]. Each fracture case costs more than 
NTD 80,000 for initial treatment in Taiwan [6]. Mostly, surgical intervention is indicated, including open reduction internal fixation or bipolar hemiarthroplasty. After that, family support and rehabilitation are also needed. Therefore, early diagnosis and prevention are vital to healthcare systems [7].

Based on the definition from IOF, osteoporosis is diagnosed as a hip-bone mineral density (BMD) T-score of -2.5 or lower. Therefore, the value of bone mass is an objective target to prevent fracture because low bone mass is a strong risk factor [8]. For aged societies, such as Taiwan, increasing attention has been paid to osteoporosis over the years [9]. According to statistics from the 2005 to 2008 National Nutrition Survey in Taiwan, the prevalence of osteoporosis was $23.9 \%$ and $38.3 \%$ for men and women aged over 50 years, respectively [10]. Osteoporosis involves genetic, nutrient, socioeconomic status (SES), and behavioral factors. By the National Health and Nutrition Examination Survey, low SES is associated with risk factors for osteoporosis [11]. People with low SES are unaware of BMD status and are delayed for initiation of medical nutrient therapy after bone-density screening $[12,13]$.The influence of behavioral factors, such as physical activity, smoking, and alcohol consumption, on bone health has been studied [14-16].

Finally, osteoporosis also elevates hip fracture risk. Besides falling, which is the major direct cause of hip fracture, hip fracture is also related to other risk factors, such as physical inactivity, low dietary calcium intake, diabetes, and Caucasian race [17-20]. Nevertheless, not all these factors are strongly associated with osteoporosis based on limited evidence. Previous studies did not find consistent association between diabetes and BMD [21-23]. There is also no absolute inverse correlation between hip fracture risk or incidence of osteoporosis and increase in dietary calcium intake, but very low calcium intake is indeed connected to a higher rate of fracture [24]. Though the Asian population has a lower BMD, lower incidence of hip fractures was found compared to Whites in the United States [25]. After a decade, hip geometry was proven as an independent factor contributing to hip fracture [26]. Among these factors, physical inactivity is consistently proven as a modifiable risk factor for either osteoporosis or bone fracture $[27,28]$. Exercise has already been proven and widely recommended for improving bone health [15]. Mechanical stimuli, such as muscle forces and ground reaction forces, increase the density and strength of bone minerals. Strength exercises prevent age-related or post-menopause-related bone loss [29-31]. Most importantly, intensity is emphasized. Walking is not as effective as resistance training alone or in combination with impact-loading activities in osteoporosis prevention [30,31]. Walking only provides a modest increase in the load on the skeleton above gravity. In addition to strength or aerobic exercise, a balance training program is also beneficial for women with established osteoporosis by reducing the incidence of falling [32].

However, previous studies have focused on specific groups, including older adults and postmenopausal women, who are at high risk for osteoporosis. Different from those studies, young adults (35-49 year old) were also recruited to our study. Among this age group, a very low percentage of people fulfill the criteria of osteoporosis [33]. These young patients usually suffer from chronic disorders or take long-term medication affecting bone metabolism, such as endocrine disorders, thalassemia, glucocorticoid, etc. Less commonly, genetic or idiopathic conditions can be found [34,35]. Patients' conditions or comorbidities can directly or indirectly affect their exercise capability and intensity. Whether the exercise is resistance training or not, the aim of this study was to investigate if the general population with a habit of regular exercise has a lower incidence of osteoporosis after propensity score matching through the Taiwan Biobank (TWB).

\section{Materials and Methods}

\subsection{Setting and Participants}

The study was approved by the Institutional Review Board of Chang Gung Memorial Hospital (IRB No. 201800396B0). The IRB also approved the waiver of the participants' consent. This research was based on the largest national biobank, Taiwan Biobank, collect- 
ing health data, questionnaires, and biological specimens, released through de-identified personal information. A total of 3320 individuals were recruited to our study cohort.

\subsection{Study Design and Data Collection}

The participants were included in the present study if they met the following criteria: (1) participants aged 35-70 years and (2) participants underwent dual-energy $X$-ray absorptiometry (DXA) for BMD measurement. Individuals with a history of cancer were excluded from the study. The questionnaire-based information included epidemiological characteristics, systemic disease, symptoms, lifestyle, social status, and nutritional factors (dietary and use of mineral or vitamin supplements). As part of the questionnaire, participants were also asked if they exercised regularly. Regular exercise was defined as a participant having an amount of exercise activity over 30 min each time, at least 3 times per week. A current alcohol drinker was defined as drinking $150 \mathrm{~mL}$ of alcohol per week for more than six months. Participants who smoked for more than six months were defined as voluntary smokers.

Participants were then divided into two groups for comparison according to their T-scores. The osteoporosis group was defined as a T-score $\leq-2.5$. Participants with a T-score $>-2.5$ were put in the non-osteoporosis group. A total of 1660 participants with osteoporosis diagnosed by DXA were first retrieved from Taiwan Biobank. After that, another 1660 participants without osteoporosis were randomly selected from the database following the same sex ratio as osteoporosis group. Finally, 3320 individuals underwent analysis.

\subsection{Taiwan Biobank (TWB)}

The TWB is conducted by the Taiwanese government and focuses on providing researchers with collaboration opportunities [36]. Several cohorts are included for population diversity, and the database is updated gradually, including a large-scale community-based cohort and hospital-based cohorts. The community-based cohort study was planned to recruit 200,000 volunteers between 30 and 70 years of age with no history of cancer. Until July 2021, 148,567 volunteer participants were recruited from three collecting sites evenly allocated in northern, southern, and eastern Taiwan. In contrast, the hospital-based cohort study aimed to recruit 100,000 patients with the most common chronic diseases in Taiwan, including lung, breast, oral cavity and colorectal cancers, hepatitis, cardiovascular disease, diabetes, chronic kidney disease, stroke, Alzheimer's disease, endometriosis, and asthma, from cooperating medical centers. Thus far, 7306 volunteer participants have been included in the project. Each subject in the TWB signed an approved informed consent form. Data collection was performed according to relevant guidelines and regulations. Apart from blood samples and physical examination, TWB researchers also conducted face-to-face interviews with participants and completed a structured questionnaire on personal information and lifestyle factors. According to official statistics, a total of 121,004 valid and available samples are in TWB currently. For demographic characteristics, the age group of 50-59 makes up the largest proportion of the age group distribution, accounting for $29.92 \%$ of the total. The male-to-female ratio is 1:1.8. In all, $57.94 \%$ subjects have studied in college, including those who already graduated. Overall, $60.49 \%$ female participants have habit of exercising, and $57.69 \%$ males do.

\subsection{BMD Measurement}

DXA scans of the central skeleton at the lumbar vertebrae were used to measure the BMD for the TWB as the gold-standard method by World Health Organization. In this case, the participants with metal lumbar implant, severe joint degenerative disease, and atherosclerosis of aorta were advised not to receive the examination. In addition to diagnosing osteoporosis, it has the advantage of assessing patients' risk of fracture and monitoring response to treatment. Although some participants' BMD was also measured 
using quantitative ultrasound (QUS) devices because of easy administration and lower price, this type of measurement correlated poorly with central DXA [37].

\subsection{Statistical Analysis}

All statistical analyses were performed using SPSS version 22.0 software (IBM Corp., Armonk, NY, USA), and the significance level for the two-tailed tests was set at 0.05. Data were tabulated as mean and standard deviation (SD) for quantitative variables and as absolute numbers. The Kolmogorov-Smirnov test was used to analyze the distribution of the variables. Categorical variables are presented as numbers. Comparisons between categorical groups were made using the chi-square analysis. The variables of demographic and clinical characteristics, SES, and lifestyles, which were significantly different between osteoporosis group $(n=1660)$ and non-osteoporosis group $(n=1660)$, were used in the binary logistic regression model to generate the propensity score. Using the propensity score, each group of patients was matched in a 1:1 ratio using Greedy's nearest neighbor method (caliper of 0.2). Logistic regression analysis was performed to estimate the odds ratios (ORs) and 95\% confidence intervals (CIs) of osteoporosis in relation to clinical characteristics, such as regular exercise and weight-control behavior.

\section{Results}

Of the 3320 subjects who received DXA for BMD measurement, 1660 people were diagnosed with osteoporosis according to a T-score $\leq-2.5$. The remaining 1660 people were referred to the non-osteoporosis group. To minimize the differences in these two groups' baseline demographic and clinical characteristics and lifestyle, propensity score matching was used. The variables that we included in propensity score matching were age, weight, fat body rate, waistline, hipline, diabetes, vertigo, cataract, heart rate, red blood cell (RBC) count, hemoglobin ( $\mathrm{Hb})$, hematocrit (Hct), fasting blood sugar, and uric acid. For SES and lifestyle, we also included education level, income, marital status, residence, and smoking in our propensity score-matching procedure. In total, 2214 people were finally analyzed after matching. Table 1 and Figure 1 show a comparison of demographic data and comorbidities between the two groups. The average age of the participants in the osteoporosis group was significantly higher. Participants whose T-score $>-2.5$ had higher body weight, fat body rate, waistline, and hipline. No significant differences were observed for most comorbidities. The prevalence of diabetes mellitus (DM) in the osteoporosis group was $7.5 \%$, which was significantly lower than that in the non-osteoporosis group. Clinical symptoms, including vertigo, joint stiffness, neck pain, sciatic pain, and headache, were present in $6.1 \%, 28.1 \%, 31.9 \%, 9.8 \%$, and $20.5 \%$ of the cases, respectively. Although the prevalence of musculoskeletal problems was high, no significant difference between the two groups was observed.

Heart rate, blood pressure, and blood tests, including complete blood count and clinical biochemistry, were measured, and the results are shown in Table 2 . The osteoporosis group had a faster heart rate, lower hemoglobin, and lower hematocrit $(p=<0.001,<0.001$, and $<0.001$, respectively). Regarding the lipid profile, people without osteoporosis had lower low-density lipoprotein levels although the data of both groups were within the acceptable normal range. The osteoporosis group also had a lower average level of uric acid in the plasma.

In Table 3, social status, including income, education, marital status, and place of residence, were also compared. Income and education levels showed significant differences. A higher percentage of individuals without osteoporosis had a college degree $(41.3 \%)$ than those with osteoporosis (35.8\%). The place of residence was related to the T-score. Participants with osteoporosis included a higher proportion of people living in northern Taiwan where urbanization is greater. 
Table 1. Demographic characteristics of body compositions and medical history.

\begin{tabular}{|c|c|c|c|c|c|c|}
\hline \multirow[b]{2}{*}{ Variable } & \multicolumn{3}{|c|}{ Before Matching } & \multicolumn{3}{|c|}{ After Matching } \\
\hline & $\begin{array}{l}\text { T-Score }>-2.5 \\
(n=1660)\end{array}$ & $\begin{array}{c}\text { T-Score } \leq-2.5 \\
(n=1660)\end{array}$ & $\begin{array}{c}p- \\
\text { Value }\end{array}$ & $\begin{array}{c}\text { T-Score }>-2.5 \\
\quad(n=1107)\end{array}$ & $\begin{array}{c}\text { T-Score } \leq-2.5 \\
(n=1107)\end{array}$ & $\begin{array}{c}p- \\
\text { Value }\end{array}$ \\
\hline Age & $57.08 \pm 7.13$ & $58.14 \pm 7.03$ & $<0.001$ & $57.61 \pm 6.87$ & $57.79 \pm 7.03$ & 0.553 \\
\hline Male sex (\%) & 665(40.1) & $665(40.1)$ & $>0.999$ & 435(39.3) & $430(38.8)$ & 0.862 \\
\hline Height (cm) & $160.81 \pm 8.06$ & $160.28 \pm 8.60$ & 0.070 & $160.42 \pm 7.91$ & $160.56 \pm 8.52$ & 0.677 \\
\hline Weight (kg) & $64.07 \pm 11.01$ & $61.14 \pm 11.42$ & $<0.001$ & $62.47 \pm 10.35$ & $62.14 \pm 11.51$ & 0.478 \\
\hline Fat body rate (\%) & $28.91 \pm 7.67$ & $27.62 \pm 7.26$ & $<0.001$ & $28.20 \pm 7.24$ & $28.17 \pm 7.22$ & 0.931 \\
\hline Waistline & $85.64 \pm 9.37$ & $83.85 \pm 9.41$ & $<0.001$ & $84.46 \pm 8.77$ & $84.59 \pm 9.38$ & 0.733 \\
\hline Hipline & $96.65 \pm 6.59$ & $95.02 \pm 6.33$ & $<0.001$ & $95.79 \pm 6.21$ & $95.75 \pm 6.25$ & 0.862 \\
\hline Allergic history (\%) & 165(9.9) & 177(10.7) & 0.530 & $109(9.8)$ & $112(10.1)$ & 0.887 \\
\hline Arthritis (\%) & $127(7.7)$ & $139(8.4)$ & 0.482 & $82(7.4)$ & $89(8.0)$ & 0.633 \\
\hline Gout (\%) & $89(5.4)$ & $70(4.2)$ & 0.143 & $55(5.0)$ & $51(4.6)$ & 0.765 \\
\hline Asthma (\%) & $55(3.3)$ & $53(3.2)$ & 0.922 & $33(3.0)$ & $31(2.8)$ & 0.899 \\
\hline Rheumatic heart (\%) & $47(2.8)$ & $52(3.1)$ & 0.683 & $27(2.4)$ & $38(3.4)$ & 0.208 \\
\hline CAD $(\%)$ & $33(2.0)$ & $40(2.4)$ & 0.478 & 19(1.7) & $29(2.6)$ & 0.189 \\
\hline Arrhythmia (\%) & $104(6.3)$ & $97(5.8)$ & 0.662 & $76(6.9)$ & $72(6.5)$ & 0.799 \\
\hline Hyperlipidemia (\%) & $159(9.6)$ & $156(9.4)$ & 0.906 & $113(10.2)$ & $94(8.5)$ & 0.189 \\
\hline Hypertension (\%) & 293(17.7) & 301(18.1) & 0.751 & 193(17.4) & 203(18.3) & 0.618 \\
\hline Diabetes mellitus (\%) & $158(9.5)$ & $125(7.5)$ & 0.047 & $99(8.9)$ & $96(8.7)$ & 0.881 \\
\hline Peptic ulcer (\%) & $302(18.2)$ & $301(18.1)$ & $>0.999$ & $200(18.1)$ & 196(17.7) & 0.868 \\
\hline GERD (\%) & $209(12.6)$ & $223(13.4)$ & 0.503 & $138(12.5)$ & 144(13.0) & 0.750 \\
\hline IBS (\%) & $35(2.1)$ & $36(2.2)$ & $>0.999$ & $23(2.1)$ & $25(2.3)$ & 0.884 \\
\hline Depression disorder (\%) & $66(4.0)$ & $64(3.9)$ & 0.929 & $52(4.7)$ & $39(3.5)$ & 0.199 \\
\hline Gallbladder stone (\%) & $88(5.3)$ & $105(6.3)$ & 0.235 & $59(5.3)$ & $70(6.3)$ & 0.364 \\
\hline Kidney stone (\%) & $121(7.3)$ & $152(9.2)$ & 0.058 & $95(8.6)$ & $93(8.4)$ & 0.939 \\
\hline Vertigo (\%) & $70(4.2)$ & $102(6.1)$ & 0.015 & $58(5.2)$ & $49(4.4)$ & 0.428 \\
\hline Joint stiffness (\%) & $421(25.4)$ & $467(28.1)$ & 0.078 & $272(24.6)$ & $304(27.5)$ & 0.133 \\
\hline Neck pain $(\%)$ & $501(30.2)$ & $529(31.9)$ & 0.311 & $342(30.9)$ & $342(30.9)$ & $>0.999$ \\
\hline Sciatic pain (\%) & $164(9.9)$ & $163(9.8)$ & $>0.999$ & $104(9.4)$ & $112(10.1)$ & 0.616 \\
\hline Headache $(\%)$ & $304(18.3)$ & $341(20.5)$ & 0.114 & $206(18.6)$ & $221(20.0)$ & 0.451 \\
\hline Cataract $(\%)$ & $234(14.1)$ & $294(17.7)$ & 0.005 & $184(16.6)$ & $168(15.2)$ & 0.383 \\
\hline Glaucoma (\%) & $41(2.5)$ & $33(2.0)$ & 0.411 & $23(2.1)$ & $23(2.1)$ & $>0.999$ \\
\hline Dry-eye syndrome (\%) & $238(14.3)$ & $263(15.8)$ & 0.245 & $164(14.8)$ & $171(15.4)$ & 0.722 \\
\hline Retinal detachment (\%) & $33(2.0)$ & $48(2.9)$ & 0.115 & $26(2.3)$ & $34(3.1)$ & 0.360 \\
\hline Myodesopsia (\%) & $258(15.5)$ & $277(16.7)$ & 0.396 & $178(16.1)$ & $181(16.4)$ & 0.908 \\
\hline
\end{tabular}

All values as mean SD or number and percent. CAD, coronary artery disease; GERD, gastroesophageal reflux disease.

A comparison of lifestyle habits, such as eating habits, smoking, alcohol drinking, dietary patterns, and exercise, is shown in Table 4 . Over $60 \%$ participants cooked at home, and the frequency of eating out did not affect the incidence of osteoporosis. Details of dietary supplements were not collected using the structure questionnaire. There was no significant difference in the pattern of dietary supplement use between the two groups. Less than half of the patients who exercised regularly had osteoporosis. Osteoporosis occurred significantly less in individuals with regular exercise and weight control than in those who did not. After propensity score matching, individuals exercising regularly or controlling body weight had a lower risk of osteoporosis (Table 5, odds ratio: 0.709 and 0.753 , respectively). 

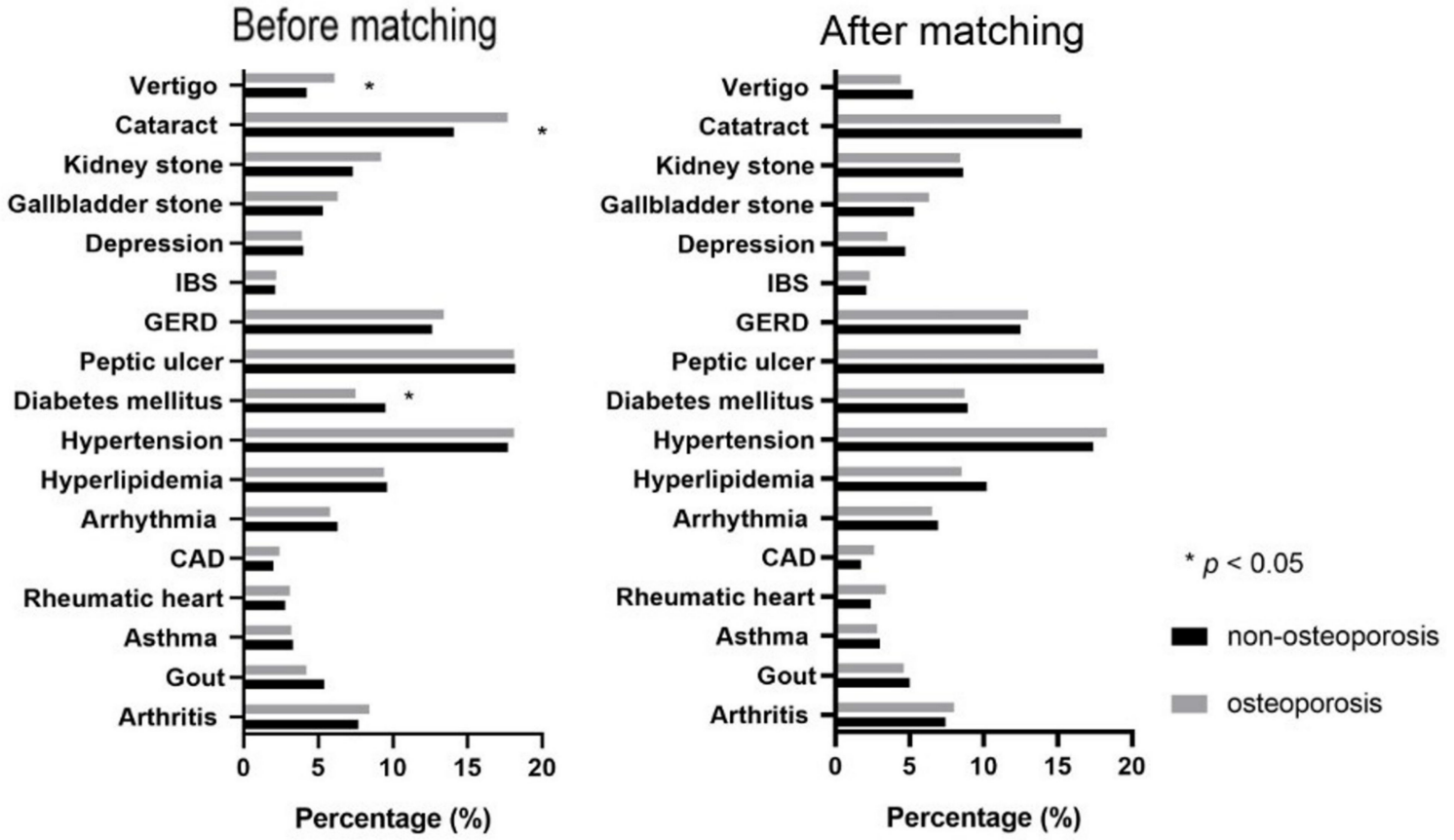

Figure 1. Comorbidities in osteoporosis and non-osteoporosis group.

Table 2. Physiological parameters and biochemistry data.

\begin{tabular}{|c|c|c|c|c|c|c|}
\hline \multirow[b]{2}{*}{ Variable } & \multicolumn{3}{|c|}{ Before Matching } & \multicolumn{3}{|c|}{ After Matching } \\
\hline & $\begin{array}{c}\text { T-Score }>-2.5 \\
\quad(n=1660)\end{array}$ & $\begin{array}{c}\text { T-Score } \leq-2.5 \\
(n=1660)\end{array}$ & $\begin{array}{c}p- \\
\text { Value }\end{array}$ & $\begin{array}{l}\text { T-Score }>-2.5 \\
\quad(n=1107)\end{array}$ & $\begin{array}{c}\text { T-Score } \leq-2.5 \\
\quad(n=1107)\end{array}$ & $\begin{array}{c}p- \\
\text { Value }\end{array}$ \\
\hline $\mathrm{SBP}(\mathrm{mmHg})$ & $122.01 \pm 17.48$ & $123.02 \pm 18.78$ & 0.110 & $122.77 \pm 17.98$ & $122.27 \pm 18.97$ & 0.532 \\
\hline DBP (mmHg) & $73.15 \pm 10.70$ & $73.40 \pm 10.96$ & 0.500 & $73.21 \pm 10.85$ & $72.99 \pm 10.91$ & 0.629 \\
\hline Heart rate & $34.90 \pm 4.59$ & $35.58 \pm 4.83$ & $<0.001$ & $35.32 \pm 4.73$ & $35.09 \pm 4.58$ & 0.239 \\
\hline $\mathrm{RBC}$ & $4.77 \pm 0.51$ & $4.68 \pm 0.51$ & $<0.001$ & $4.73 \pm 0.51$ & $4.71 \pm 0.49$ & 0.592 \\
\hline WBC & $5.75 \pm 1.46$ & $5.81 \pm 1.57$ & 0.226 & $5.77 \pm 1.50$ & $5.70 \pm 1.44$ & 0.249 \\
\hline $\mathrm{Hb}$ & $14.00 \pm 1.46$ & $13.79 \pm 1.47$ & $<0.001$ & $13.89 \pm 1.48$ & $13.87 \pm 1.43$ & 0.823 \\
\hline Hct & $43.40 \pm 4.33$ & $42.78 \pm 4.28$ & $<0.001$ & $43.03 \pm 4.38$ & $43.06 \pm 4.17$ & 0.855 \\
\hline Plt & $229.18 \pm 55.06$ & $228.35 \pm 54.89$ & 0.663 & $229.63 \pm 55.87$ & $227.70 \pm 54.20$ & 0.409 \\
\hline HbA1c (\%) & $5.99 \pm 0.96$ & $5.96 \pm 0.91$ & 0.268 & $5.97 \pm 0.89$ & $5.98 \pm 0.93$ & 0.794 \\
\hline $\begin{array}{l}\text { Fasting blood sugar } \\
\text { (mg/dL) }\end{array}$ & $101.10 \pm 26.65$ & $99.33 \pm 24.38$ & 0.047 & $100.54 \pm 25.14$ & $99.88 \pm 24.97$ & 0.536 \\
\hline Total cholesterol (mg/dL) & $200.76 \pm 36.95$ & $200.11 \pm 36.90$ & 0.615 & $200.21 \pm 36.99$ & $200.54 \pm 36.36$ & 0.832 \\
\hline $\mathrm{TG}(\mathrm{mg} / \mathrm{dL})$ & $120.21 \pm 86.33$ & $123.99 \pm 102.88$ & 0.251 & $119.57 \pm 75.25$ & $118.61 \pm 79.84$ & 0.770 \\
\hline $\operatorname{HDL}(\mathrm{mg} / \mathrm{dL})$ & $54.14 \pm 13.73$ & $54.90 \pm 13.99$ & 0.119 & $54.62 \pm 14.38$ & $54.85 \pm 13.47$ & 0.693 \\
\hline LDL (mg/dL) & $125.53 \pm 32.10$ & $123.23 \pm 32.08$ & 0.039 & $124.41 \pm 32.49$ & $124.57 \pm 32.46$ & 0.906 \\
\hline Total bilirubin (g/dL) & $0.69 \pm 0.29$ & $0.68 \pm 0.27$ & 0.248 & $0.69 \pm 0.30$ & $0.69 \pm 0.27$ & 0.934 \\
\hline Albumin (g/dL) & $4.54 \pm 0.24$ & $4.52 \pm 0.23$ & 0.008 & $4.53 \pm 0.24$ & $4.53 \pm 0.23$ & 0.510 \\
\hline GPT $(\mathrm{U} / \mathrm{L})$ & $25.24 \pm 20.33$ & $24.13 \pm 17.42$ & 0.092 & $25.07 \pm 20.26$ & $24.06 \pm 16.11$ & 0.192 \\
\hline$\alpha$-fetoprotein $(\mathrm{U} / \mathrm{L})$ & $3.35 \pm 3.83$ & $3.48 \pm 3.31$ & 0.312 & $3.42 \pm 4.55$ & $3.37 \pm 1.75$ & 0.703 \\
\hline$\gamma-\mathrm{GT}(\mathrm{U} / \mathrm{L})$ & $25.63 \pm 25.62$ & $28.32 \pm 52.69$ & 0.062 & $26.19 \pm 28.05$ & $25.68 \pm 27.43$ & 0.662 \\
\hline BUN (mg/dL) & $14.36 \pm 3.93$ & $14.17 \pm 4.87$ & 0.223 & $14.38 \pm 3.88$ & $14.37 \pm 4.46$ & 0.957 \\
\hline Creatinine $(\mathrm{mg} / \mathrm{dL})$ & $0.75 \pm 0.21$ & $0.74 \pm 0.39$ & 0.169 & $0.75 \pm 0.21$ & $0.74 \pm 0.36$ & 0.333 \\
\hline Uric acid (mg/dL) & $5.72 \pm 1.37$ & $5.47 \pm 1.39$ & $<0.001$ & $5.60 \pm 1.35$ & $5.55 \pm 1.38$ & 0.345 \\
\hline $\begin{array}{l}\text { Urine microalbumin } \\
\qquad(\mathrm{mg} / \mathrm{dL})\end{array}$ & $32.86 \pm 195.62$ & $34.07 \pm 142.98$ & 0.838 & $28.14 \pm 108.75$ & $37.40 \pm 163.67$ & 0.117 \\
\hline
\end{tabular}

All values as mean SD or number and percent. SBP, systolic blood pressure; DBP, diastolic blood pressure $\mathrm{RBC}$, red blood cell; WBC, white blood cell; Hb, hemoglobin; Hct, hematocrit; Plt, platelet, TG, triglycerides, HDL, high-density lipoprotein; LDL, low-density lipoprotein; GPT, glutamate pyruvate transaminase; $\gamma$-GT, $\gamma$-glutamyl transferase. 
Table 3. Socioeconomic, residency, and educational status.

\begin{tabular}{|c|c|c|c|c|c|c|}
\hline \multirow[b]{2}{*}{ Variable } & \multicolumn{3}{|c|}{ Before Matching } & \multicolumn{3}{|c|}{ After Matching } \\
\hline & $\begin{array}{c}\text { T-Score }>-2.5 \\
\quad(n=1660)\end{array}$ & $\begin{array}{c}\text { T-Score } \leq-2.5 \\
(n=1660)\end{array}$ & $\begin{array}{c}p- \\
\text { Value }\end{array}$ & $\begin{array}{c}\text { T-Score }>-2.5 \\
\quad(n=1107)\end{array}$ & $\begin{array}{c}\text { T-Score } \leq-2.5 \\
(n=1107)\end{array}$ & $\begin{array}{c}p- \\
\text { Value }\end{array}$ \\
\hline Income (per month) & $5.21 \pm 3.44$ & $4.97 \pm 3.29$ & 0.045 & $5.03 \pm 3.41$ & $5.10 \pm 3.34$ & 0.600 \\
\hline Education & & & $<0.001$ & & & 0.478 \\
\hline College & $685(41.3)$ & $595(35.8)$ & & $429(38.8)$ & $440(39.7)$ & \\
\hline Senior high school & $541(32.6)$ & $561(33.8)$ & & $367(33.2)$ & $365(33.0)$ & \\
\hline Junior high school & $200(12.0)$ & $228(13.7)$ & & $139(12.6)$ & $127(11.5)$ & \\
\hline Elementary school & $218(13.1)$ & $254(15.3)$ & & $158(14.3)$ & $160(14.5)$ & \\
\hline Illiteracy & $16(1.0)$ & $22(1.3)$ & & $14(1.3)$ & $15(1.4)$ & \\
\hline Marital status & & & $<0.001$ & & & 0.239 \\
\hline Married & $1338(80.6)$ & $1273(76.7)$ & & $870(78.6)$ & $867(78.3)$ & \\
\hline Single & $64(3.9)$ & $86(5.2)$ & & $51(4.6)$ & $48(4.3)$ & \\
\hline Divorced/separated/widowed & $258(15.5)$ & 301(18.1) & & $186(16.8)$ & 192(17.3) & \\
\hline Residence (\%) & & & $<0.001$ & & & 0.855 \\
\hline Northern Taiwan & $320(19.3)$ & $392(23.6)$ & & $226(20.4)$ & $248(22.4)$ & \\
\hline Central Taiwan & $401(24.2)$ & $439(26.4)$ & & $278(25.1)$ & $273(24.7)$ & \\
\hline Southern Taiwan & $911(54.9)$ & $797(48.0)$ & & $584(52.8)$ & $568(51.3)$ & \\
\hline Eastern Taiwan & $28(1.7)$ & $32(1.9)$ & & $19(1.7)$ & $18(1.6)$ & \\
\hline
\end{tabular}

All values as mean SD or number and percent. CAD, coronary artery disease; GERD, gastroesophageal reflux disease.

Table 4. Dietary habits, healthy behavior, and healthcare seeking.

\begin{tabular}{|c|c|c|c|c|c|c|}
\hline \multirow[b]{2}{*}{ Variable } & \multicolumn{3}{|c|}{ Before Matching } & \multicolumn{3}{|c|}{ After Matching } \\
\hline & $\begin{array}{c}\text { T-Score }>-2.5 \\
\quad(n=1660)\end{array}$ & $\begin{array}{c}\text { T-Score } \leq-2.5 \\
(n=1660)\end{array}$ & $\begin{array}{c}p- \\
\text { Value }\end{array}$ & $\begin{array}{c}\text { T-Score }>-2.5 \\
\quad(n=1107)\end{array}$ & $\begin{array}{l}\text { T-Score } \leq-2.5 \\
\quad(n=1107)\end{array}$ & $\begin{array}{c}p- \\
\text { Value }\end{array}$ \\
\hline Cook at home & $1034(62.3)$ & 1001(60.3) & 0.254 & $679(61.3)$ & 685(61.9) & 0.827 \\
\hline Eat late-night supper & $378(22.8)$ & $425(25.6)$ & 0.062 & $252(22.8)$ & $276(24.9)$ & 0.251 \\
\hline Eat out & & & 0.579 & & & 0.963 \\
\hline 1 meal per day & 290(17.5) & 264(15.9) & & $183(16.5)$ & 173(15.6) & \\
\hline 2-3 meal per day & 197(11.9) & $219(13.2)$ & & 132(11.9) & $142(12.8)$ & \\
\hline $1-3$ meals per week & $424(25.5)$ & $426(25.7)$ & & $269(24.3)$ & $280(25.3)$ & \\
\hline 4-6 meals per week & $118(7.1)$ & $112(6.7)$ & & $78(7.0)$ & $77(7.0)$ & \\
\hline $1-3$ meals per month & $499(30.1)$ & $489(29.5)$ & & $347(31.3)$ & $338(30.5)$ & \\
\hline None & $132(8.0)$ & $150(9.0)$ & & $98(8.9)$ & $97(8.8)$ & \\
\hline Source of drinking-water & & & 0.637 & & & 0.700 \\
\hline Well water & $6(0.4)$ & $11(0.7)$ & & $4(0.4)$ & $9(0.8)$ & \\
\hline Tap water & $256(15.4)$ & $271(16.3)$ & & $168(15.2)$ & $171(15.4)$ & \\
\hline Purified water & $1191(71.7)$ & $1161(69.9)$ & & $792(71.5)$ & $789(71.3)$ & \\
\hline Mineral water & $75(4.5)$ & $77(4.6)$ & & $55(5.0)$ & $50(4.5)$ & \\
\hline Others & $132(8.0)$ & $140(8.4)$ & & $88(7.9)$ & $88(7.9)$ & \\
\hline Drink tea & $504(30.4)$ & $510(30.7)$ & 0.851 & $318(28.7)$ & $346(31.3)$ & 0.210 \\
\hline Drink coffee & $545(32.8)$ & $531(32.0)$ & 0.630 & $361(32.6)$ & $353(31.9)$ & 0.750 \\
\hline Vegetarian & & & 0.373 & & & 0.474 \\
\hline Used to be & $65(3.9)$ & $61(3.7)$ & & $44(4.0)$ & $34(3.1)$ & \\
\hline Yes & $82(4.9)$ & $100(6.0)$ & & $60(5.4)$ & $65(5.9)$ & \\
\hline No & 1513(91.1) & 1499(90.3) & & 1003(90.6) & 1008(91.1) & \\
\hline
\end{tabular}


Table 4. Cont.

\begin{tabular}{|c|c|c|c|c|c|c|}
\hline \multirow[b]{2}{*}{ Variable } & \multicolumn{3}{|c|}{ Before Matching } & \multicolumn{3}{|c|}{ After Matching } \\
\hline & $\begin{array}{c}\text { T-Score }>-2.5 \\
\quad(n=1660)\end{array}$ & $\begin{array}{c}\text { T-Score } \leq-2.5 \\
\quad(n=1660)\end{array}$ & $\begin{array}{c}p- \\
\text { Value }\end{array}$ & $\begin{array}{l}\text { T-Score }>-2.5 \\
\quad(n=1107)\end{array}$ & $\begin{array}{c}\text { T-Score } \leq-2.5 \\
\quad(n=1107)\end{array}$ & $\begin{array}{c}p- \\
\text { Value }\end{array}$ \\
\hline Drink alcohol & & & 0.996 & & & 0.321 \\
\hline Quit & $58(3.5)$ & $59(3.6)$ & & $38(3.4)$ & $35(3.2)$ & \\
\hline Frequently & $110(6.6)$ & $110(6.6)$ & & $73(6.6)$ & $57(5.1)$ & \\
\hline No or rarely & 1492(89.9) & $1491(89.8)$ & & $996(90.0)$ & 1015(91.7) & \\
\hline Voluntary smoking & $107(6.4)$ & $196(11.8)$ & $<0.001$ & $92(8.3)$ & $83(7.5)$ & 0.529 \\
\hline Involuntary smoking & $159(9.6)$ & $179(10.8)$ & 0.275 & $99(8.9)$ & 103(9.3) & 0.825 \\
\hline Chewing betel nut & $22(1.3)$ & $33(2.0)$ & 0.174 & $18(1.6)$ & $14(1.3)$ & 0.593 \\
\hline Substance dependence & $27(1.6)$ & $31(1.9)$ & 0.691 & $16(1.4)$ & $19(1.7)$ & 0.734 \\
\hline Taking dietary Supplement & & & 0.989 & & & 0.638 \\
\hline Irregularly & $367(22.1)$ & $365(22.0)$ & & $247(22.3)$ & $239(21.6)$ & \\
\hline Regularly & $601(36.2)$ & $605(36.4)$ & & $406(36.7)$ & 392(35.4) & \\
\hline None & 692(41.7) & $690(41.6)$ & & $454(41.0)$ & $476(43.0)$ & \\
\hline $\begin{array}{c}\text { Behavior of seeking } \\
\text { Healthcare }\end{array}$ & & & 0.342 & & & 0.456 \\
\hline $\begin{array}{c}\text { Visit Chinese traditional } \\
\text { Doctor }\end{array}$ & 156(9.4) & 161(9.7) & & 108(9.8) & 106(9.6) & \\
\hline Visit doctor & $975(58.7)$ & $957(57.7)$ & & $649(58.6)$ & $652(58.9)$ & \\
\hline Go to pharmacy & $123(7.4)$ & $116(7.0)$ & & $76(6.9)$ & $66(6.0)$ & \\
\hline Folk medicine & $28(1.7)$ & $27(1.6)$ & & $19(1.7)$ & $18(1.6)$ & \\
\hline Observation & $226(13.6)$ & $208(12.5)$ & & $158(14.3)$ & $142(12.8)$ & \\
\hline Others & $226(13.6)$ & $208(12.5)$ & & $158(14.3)$ & $142(12.8)$ & \\
\hline Regular exercise & $963(58.0)$ & $820(49.4)$ & $<0.001$ & $658(59.4)$ & $564(50.9)$ & $<0.001$ \\
\hline Weight control & $826(49.8)$ & $670(40.4)$ & $<0.001$ & $547(49.4)$ & $469(42.4)$ & 0.001 \\
\hline
\end{tabular}

All values as mean SD or number and percent. CAD, coronary artery disease; GERD, gastroesophageal reflux disease.

Table 5. Individual risk of osteoporosis for T-Score $\leq-2.5$.

\begin{tabular}{ccc}
\hline & OR & $\mathbf{9 5 \%}$ CI of OR \\
\hline Regular exercise & 0.709 & $0.599-0.839$ \\
Weight control & 0.753 & $0.636-0.890$ \\
\hline
\end{tabular}

OR, odds ratio; $\mathrm{CI}$, confidence interval.

\section{Discussion}

Any reason that leads to an imbalance between bone resorption and formation causes osteoporosis. Factors for osteoporosis can be classified as modifiable or non-modifiable. Reducing modifiable risk factors, such as inadequate nutritional absorption, lack of physical activity, weight loss, cigarette smoking, alcohol consumption, air pollution, or stress, has been proven to prevent osteoporosis [38]. In this study, propensity score matching was used for known non-modifiable factors and a few modifiable factors for osteoporosis. As a result, regular exercise and weight control are both independent factors in preventing osteoporosis.

Evidence from many RCTs and meta-analyses showed that exercise training, such as weight-bearing impact exercise and progressive resistance training, can promote the bone health of children and adolescents, pre- and postmenopausal women, and older men [3942]. In this study, the exercises were not limited to resistance or weight-bearing training. On the contrary, "exercise" was defined as activities during which the participant breathed deeply or perspired in the questionnaire. An open-ended question was asked to determine the exercises done by the participants. Most people chose aerobic exercise as a routine exercise. However, the participants could write down a maximum of three exercises.

Therefore, it was difficult to further investigate which exercise was potentially effective in preventing osteoporosis. Although regular exercise and body weight control were shown to be protective factors for osteoporosis, the regularity and self-discipline were not defined or further evaluated. Participants who stood on the positive side for these two questions 
cared more about their health condition than did others. As shown in Supplementary Figure S1, a variety of exercises were performed. Although most exercises were classified as weight-bearing exercises, other aerobic exercises, such as swimming, biking, and tai-chi, which specifically improve cardiovascular function, balance, and body strength, were also options for participants.

An association was found between body weight and bone mineral density though the relationship was still controversial. Jensen et al. and Villareal et al. pointed out that weight loss is associated with a decrease in BMD $[43,44]$. As obesity increases, bone mineralization increases, leading to a reduced risk of osteoporosis and related diseases [45]. A recent systematic review and meta-analysis in 2020 also found obesity as a protective factor to osteoporosis [46]. On the contrary, some studies also have indicated that low BMD is strongly associated with increased percentage body fat (PBF) in adults with obesity [47-49]. To date, the mechanism of this correlation remains unclear although several explanations have been proposed. The acceptable hypothesis is that bone mass increases to accommodate the greater mechanical load from a larger body mass. However, weight loss does not adversely affect BMD as long as the muscle is maintained with exercise training. In contrast, changes in BMD were correlated with changes in thigh muscle volume [50]. Muscle was more strongly related to BMD than fat or body weight. Maintenance of lean tissue and prevention of fat mass gain may be important for maintaining bone health. According to a longitudinal cohort study, a loss of lean tissue $(0.9 \%)$ and gain in fat $(9 \%)$ occurred concomitantly with a decrease in bone mass (1.6\%) over 5 years in men aged 25 to 96 years [51].

Type 2 diabetes mellitus is significantly associated with osteoporosis risk. A study by Lin et al. from Taiwan concluded a positive association between the presence of type 2 $\mathrm{DM}$ and the incidence of osteoporosis [52]. Another small sample, cross-sectional study revealed no significant difference in bone density between diabetic and nondiabetic subjects [53]. Nevertheless, DM was more common among participants without osteoporosis, in conflict with previous evidence. This could be explained by the fact that the comorbidities of participants were self-reported. Prediabetes could sometimes be mistaken for diabetes because we did not observe differences in $\mathrm{HbA1C}$ levels between obese individuals. According to the mean value of $\mathrm{HbA1C}$, over half of the participants are in the status of prediabetes. This might affect the statistical results. Moreover, although fasting sugar in the non-osteoporosis group was higher, clinical significance was not observed, and the test is less reliable compared to $\mathrm{HbA} 1 \mathrm{C}$ test due to human error.

In postmenopausal women, adipocytes contribute to the production of estrogen, which inhibits bone resorption by osteoclasts [54]. Increases in adipose tissue with increasing BMI in postmenopausal women result in increased estrogen production, osteoclast suppression, and a resultant increase in bone mass. Previous study also verified our findings, as it reported that people with a higher body fat rate had a lower percentage of osteoporosis $[14,54]$.

Smoking is known to cause osteoporosis and bone fracture. Cadmium contained in tobacco has been shown to play a critical role in the underlying mechanism. Smoking is therefore a major source of cadmium exposure in smokers. Wallin et al. indicated that even low cadmium exposure increases the risk of low BMD and fractures. They found that 10 pack-years of smoking could shorten the time to first hip fracture by 3\% [55]. Although the amount of smoking was not analyzed in our study, people who smoked voluntarily had a higher percentage of osteoporosis.

A recent meta-analysis reported that supplementation with calcium and vitamin D significantly reduced the risk of fractures and hip fractures by $15 \%$ and $30 \%$, respectively [56]. A prospective study in Lyon Hospital proved that combined treatment with vitamin D and calcium supplementation is beneficial for postmenopausal women [37]. However, maintaining the correct dietary calcium phosphate is also emphasized. High calcium intake can cause the calcium paradox [57]. Satisfying the vitamin D requirements is important to obtain the best response for BMD. Vitamin D supplementation, with or without calcium, 
can increase bone mineral density (BMD) and have a positive anti-fracture effect. Vitamins and minerals are the main supplements taken by people in Taiwan [58]. However, the highest consumption was for vitamin E (19.4\%), followed by vitamin C (9.7\%) in the vitamins category. The most popular item was calcium $(20.9 \%)$ in the mineral category. In people who took vitamins and minerals, only $2.1 \%$ consumed vitamin D with calcium [31]. The low usage of vitamin D plus calcium may explain why people using dietary supplements had no lower osteoporosis incidence.

A strong connection was found between socioeconomic status (SES) and health [59]. People with lower education and lower income have a greater risk of fracture [60,61]. A study based on the Korean adult population revealed that osteoporosis was more prevalent among men with a lower household income and women living in rural areas [62]. In a cohort study by Varenna et al., a protective role was played by increases in formal education [23]. An increase in educational status was associated with a significantly reduced risk of osteoporosis. Nevertheless, the study was limited because the researchers focused on a low educational level. They did not further stratify samples with more than 9 years of formal education. The results of our study showed that people with more than a high school education comprise a large proportion of osteoporosis patients. This is because the nine-year education program from primary to junior high schools started to be implemented in Taiwan since 1968. However, the percentage of osteoporosis between the high school and college education subgroups was not significantly different.

A study on osteoporosis between urban and rural areas was also conducted by Kang et al. The prevalence of osteoporosis in rural residents was higher than that in urban area residents. This tendency was also observed in our results. The urbanization level is highest in northern Taiwan, which leads to a lower percentage of osteoporosis [63].

There are some limitations to this study. Although this is a large database conducted by the Taiwanese government, over 95\% of Taiwan's population of 23.4 million consists of Han Chinese. Second, data collection for this cohort was based on a structured and rough questionnaire. Personal information and lifestyle factors were mostly self-reported. Common comorbidities except cancers were included without consideration of the nature of diseases. The severity of diseases could potentially impact osteoporosis. Moreover, the interaction between drug and osteoporosis was not considered. In addition, some of the questions were too general, imprecise, or indefinite. More than one option of exercise could be chosen by the participant, and the intensity among these exercises was also different. Therefore, the frequency and the intensity discrepancies between different exercise cannot be controlled during analysis in this study to make our result more convincing. These limitations should be noticed in future study.

\section{Conclusions}

After propensity score matching, most covariates and known risk factors were balanced. For the general population in Taiwan, regular exercise or weight-control behavior is an independent factor for lowering the incidence of osteoporosis.

Supplementary Materials: The following are available online at https:/ / www.mdpi.com/article/ 10.3390/nu14030641/s1, Figure S1: Count of different exercises (maximum of three exercises by each participant).

Author Contributions: Conceptualization, C.-Y.H. (Chih-Yi Hsu) and C.-Y.H. (Chun-Ying Huang); methodology, C.-Y.H. (Chih-Yi Hsu) and C.-H.H.; software, P.-C.C.; validation, C.-H.H. and P.-C.C.; formal analysis, P.-C.C.; investigation, C.-C.C.; resources, C.-H.H.; data curation, C.-C.C. and S.-Y.H.; writing-original draft preparation, C.-Y.H. (Chih-Yi Hsu); writing-review and editing, S.-C.W.; visualization, C.-C.C.; supervision, S.-C.W.; project administration, C.-Y.H. (Chun-Ying Huang) and C.-H.H.; funding acquisition, C.-Y.H. (Chun-Ying Huang) and S.-C.W. All authors have read and agreed to the published version of the manuscript.

Funding: This research was supported by a grant from Chang Gung Memorial Hospital (CMRPG8K1331 and CORPG8K0211) to S.-C.W. 
Institutional Review Board Statement: This study was approved by the Institutional Review Board of Kaohsiung Chang Gung Memorial Hospital (IRB number: 201800396B0).

Informed Consent Statement: Informed consent was obtained from all recruited subjects of Taiwan Biobank Project.

Data Availability Statement: The data presented in this study are available from the corresponding authors upon reasonable request.

Acknowledgments: We appreciate the statistical analyses assistance by the Biostatistics Center, Kaohsiung Chang Gung Memorial Hospital.

Conflicts of Interest: The authors declare no conflict of interest.

\section{References}

1. World Health Organization. Assessment of osteoporosis at the primary health care level. Summ. Rep. A WHO Sci. Group. WHO Geneva 2004. Available online: https://www.who.int/chp/topics/Osteoporosis.pdf (accessed on 20 January 2022).

2. Glaser, D.L.; Kaplan, F.S. Osteoporosis. Definition and clinical presentation. Spine 1997, 22, 12s-16s. [CrossRef] [PubMed]

3. Vandenbroucke, A.; Luyten, F.P.; Flamaing, J.; Gielen, E. Pharmacological treatment of osteoporosis in the oldest old. Clin. Interv Aging 2017, 12, 1065-1077. [CrossRef] [PubMed]

4. Borgström, F.; Karlsson, L.; Ortsäter, G.; Norton, N.; Halbout, P.; Cooper, C.; Lorentzon, M.; McCloskey, E.V.; Harvey, N.C.; Javaid, M.K.; et al. Fragility fractures in Europe: Burden, management and opportunities. Arch. Osteoporos. 2020, 15, 59. [CrossRef] [PubMed]

5. $\quad$ Lewiecki, E.M.; Ortendahl, J.D.; Vanderpuye-Orgle, J.; Grauer, A.; Arellano, J.; Lemay, J.; Harmon, A.L.; Broder, M.S.; Singer, A.J. Healthcare Policy Changes in Osteoporosis Can Improve Outcomes and Reduce Costs in the United States. JBMR Plus 2019, 3 , e10192. [CrossRef]

6. Hwang, J.-S.; Chan, D.-C.; Chen, J.-F.; Cheng, T.-T.; Wu, C.-H.; Soong, Y.-K.; Tsai, K.-S.; Yang, R.-S. Clinical practice guidelines for the prevention and treatment of osteoporosis in Taiwan: Summary. J. Bone Miner. Metab. 2014, 32, 10-16. [CrossRef]

7. Pisani, P.; Renna, M.D.; Conversano, F.; Casciaro, E.; Muratore, M.; Quarta, E.; Paola, M.D.; Casciaro, S. Screening and early diagnosis of osteoporosis through X-ray and ultrasound based techniques. World J. Radiol. 2013, 5, 398-410. [CrossRef] [PubMed]

8. The Royal Australian College of General Practitioners and Osteoporosis Australia. Osteoporosis Prevention, Diagnosis and Management in Postmenopausal Women and Men Over 50 Years of Age; RACGP: East Melbourne, Canada, 2017.

9. Chen, F.-P.; Huang, T.-S.; Fu, T.-S.; Sun, C.-C.; Chao, A.-S.; Tsai, T.-L. Secular trends in incidence of osteoporosis in Taiwan: A nationwide population-based study. Biomed. J. 2018, 41, 314-320. [CrossRef]

10. Yi-Chin, L.; Wen-Harn, P. Bone Mineral Density in Adults in Taiwan: Results of the Nutrition and Health Survey in Taiwan 2005-2008 (NAHSIT 2005-2008). Asia Pac. J. Clin. Nutr. 2011, 20, 283-291. [CrossRef]

11. Lyles, C.R.; Schafer, A.L.; Seligman, H.K. Income, food insecurity, and osteoporosis among older adults in the 2007-2008 National Health and Nutrition Examination Survey (NHANES). J. Health Care Poor Underserved 2014, 25, 1530-1541. [CrossRef]

12. Packard, P.T.; Heaney, R.P. Medical nutrition therapy for patients with osteoporosis. J. Am. Diet. Assoc. 1997, 97, 414-417. [CrossRef]

13. Brennan, R.M.; Wactawski-Wende, J.; Crespo, C.J.; Dmochowski, J. Factors associated with treatment initiation after osteoporosis screening. Am. J. Epidemiol. 2004, 160, 475-483. [CrossRef]

14. Bijelic, R.; Balaban, J.; Milicevic, S. Correlation of the Lipid Profile, BMI and Bone Mineral Density in Postmenopausal Women. Mater. Sociomed. 2016, 28, 412-415. [CrossRef]

15. Cheung, A.M.; Giangregorio, L. Mechanical stimuli and bone health: What is the evidence? Curr. Opin. Rheumatol. 2012, 24, 561-566. [CrossRef]

16. Zhu, K.; Prince, R.L. Lifestyle and Osteoporosis. Curr. Osteoporos. Rep. 2015, 13, 52-59. [CrossRef]

17. Lau, E.M.; Cooper, C. The epidemiology of osteoporosis. The oriental perspective in a world context. Clin. Orthop. Relat. Res. 1996, 323, 65-74. [CrossRef]

18. Ho, S.C. Body measurements, bone mass, and fractures. Does the East differ from the West? Clin. Orthop. Relat. Res. 1996, 323, 75-80. [CrossRef]

19. Nakamura, T.; Turner, C.H.; Yoshikawa, T.; Slemenda, C.W.; Peacock, M.; Burr, D.B.; Mizuno, Y.; Orimo, H.; Ouchi, Y.; Johnston, C.C., Jr. Do variations in hip geometry explain differences in hip fracture risk between Japanese and white Americans? J. Bone Miner. Res. Off. J. Am. Soc. Bone Miner. Res. 1994, 9, 1071-1076. [CrossRef]

20. Sugimoto, T.; Sato, M.; Dehle, F.C.; Brnabic, A.J.; Weston, A.; Burge, R. Lifestyle-Related Metabolic Disorders, Osteoporosis, and Fracture Risk in Asia: A Systematic Review. Value Health Reg. Issues 2016, 9, 49-56. [CrossRef]

21. Khoo, C.C.; Woo, J.; Leung, P.C.; Kwok, A.; Kwok, T. Determinants of bone mineral density in older postmenopausal Chinese women. Climacteric J. Int. Menopause Soc. 2011, 14, 378-383. [CrossRef]

22. Chen, H.-l.; Deng, L.-l.; Li, J.-f. Prevalence of Osteoporosis and Its Associated Factors among Older Men with Type 2 Diabetes. Int. J. Endocrinol. 2013, 2013, 285729. [CrossRef] 
23. Anaforoglu, I.; Nar-Demirer, A.; Bascil-Tutuncu, N.; Ertorer, M.E. Prevalence of osteoporosis and factors affecting bone mineral density among postmenopausal Turkish women with type 2 diabetes. J. Diabetes Complicat. 2009, 23, 12-17. [CrossRef] [PubMed]

24. Warensjö, E.; Byberg, L.; Melhus, H.; Gedeborg, R.; Mallmin, H.; Wolk, A.; Michaëlsson, K. Dietary calcium intake and risk of fracture and osteoporosis: Prospective longitudinal cohort study. BMJ 2011, 342, d1473. [CrossRef] [PubMed]

25. Ho, S.C.; Hsu, S.Y.; Leung, P.C.; Chan, C.; Swaminathan, R.; Fan, Y.K.; Chan, S.S. A longitudinal study of the determinants of bone mass in Chinese women aged 21 to 40 . I. Baseline association of anthropometric measurements with bone mineral density. Ann. Epidemiol. 1993, 3, 256-263. [CrossRef]

26. Yan, L.; Crabtree, N.J.; Reeve, J.; Zhou, B.; Dequeker, J.; Nijs, J.; Falch, J.A.; Prentice, A. Does hip strength analysis explain the lower incidence of hip fracture in the People's Republic of China? Bone 2004, 34, 584-588. [CrossRef]

27. Forsén, L.; Bjørndal, A.; Bjartveit, K.; Edna, T.H.; Holmen, J.; Jessen, V.; Westberg, G. Interaction between current smoking, leanness, and physical inactivity in the prediction of hip fracture. J. Bone Miner. Res. Off. J. Am. Soc. Bone Miner. Res. 1994, 9 , 1671-1678. [CrossRef]

28. LaMonte, M.J.; Wactawski-Wende, J.; Larson, J.C.; Mai, X.; Robbins, J.A.; LeBoff, M.S.; Chen, Z.; Jackson, R.D.; LaCroix, A.Z.; Ockene, J.K.; et al. Association of Physical Activity and Fracture Risk Among Postmenopausal Women. JAMA Netw. Open 2019, 2, e1914084. [CrossRef]

29. Gómez-Cabello, A.; Ara, I.; González-Agüero, A.; Casajús, J.A.; Vicente-Rodríguez, G. Effects of Training on Bone Mass in Older Adults. Sports Med. 2012, 42, 301-325. [CrossRef]

30. Zehnacker, C.H.; Bemis-Dougherty, A. Effect of Weighted Exercises on Bone Mineral Density in Post Menopausal Women A Systematic Review. J. Geriatr. Phys. Ther. 2007, 30, 79-88. [CrossRef]

31. Bolam, K.A.; van Uffelen, J.G.Z.; Taaffe, D.R. The effect of physical exercise on bone density in middle-aged and older men: A systematic review. Osteoporos. Int. 2013, 24, 2749-2762. [CrossRef]

32. Miko, I.; Szerb, I.; Szerb, A.; Bender, T.; Poor, G.J.J.o.r.m. Effect of a balance-training programme on postural balance, aerobic capacity and frequency of falls in women with osteoporosis: A randomized controlled trial. J. Rehabil. Med. 2018, 50, 542-547. [CrossRef]

33. Ferrari, S.L.; Bianchi, M.L.; Eisman, J.A.; Foldes, A.J.; Adami, S.; Wahl, D.A.; Stepan, J.; De Vernejoul, M.-C.; Kaufman, J.-M. Osteoporosis in young adults: Pathophysiology, diagnosis, and management. Osteoporos. Int. 2012, 23, 2735-2748. [CrossRef]

34. Bonjour, J.P.; Chevalley, T.; Rizzoli, R.; Ferrari, S. Gene-environment interactions in the skeletal response to nutrition and exercise during growth. Med. Sport Sci. 2007, 51, 64-80. [CrossRef]

35. Ferrari, S.; Rizzoli, R.; Slosman, D.; Bonjour, J.P. Familial resemblance for bone mineral mass is expressed before puberty. J. Clin. Endocrinol. Metab. 1998, 83, 358-361. [CrossRef]

36. Fan, C.-T.; Lin, J.-C.; Lee, C.-H. Taiwan Biobank: A project aiming to aid Taiwan's transition into a biomedical island. Pharmacogenomics 2008, 9, 235-246. [CrossRef]

37. Lu, Y.; Genant, H.K.; Shepherd, J.; Zhao, S.; Mathur, A.; Fuerst, T.P.; Cummings, S.R. Classification of Osteoporosis Based on Bone Mineral Densities. J. Bone Miner. Res. 2001, 16, 901-910. [CrossRef]

38. Pouresmaeili, F.; Kamalidehghan, B.; Kamarehei, M.; Goh, Y.M. A comprehensive overview on osteoporosis and its risk factors Ther. Clin. Risk Manag. 2018, 14, 2029-2049. [CrossRef]

39. Behringer, M.; Gruetzner, S.; McCourt, M.; Mester, J. Effects of Weight-Bearing Activities on Bone Mineral Content and Density in Children and Adolescents: A Meta-Analysis. J. Bone Miner. Res. 2014, 29, 467-478. [CrossRef]

40. Kelley, G.A.; Kelley, K.S.; Kohrt, W.M. Exercise and Bone Mineral Density in Premenopausal Women: A Meta-Analysis of Randomized Controlled Trials. Int. J. Endocrinol. 2013, 2013, 741639. [CrossRef]

41. Zhao, R.; Zhao, M.; Xu, Z. The effects of differing resistance training modes on the preservation of bone mineral density in postmenopausal women: A meta-analysis. Osteoporos. Int. 2015, 26, 1605-1618. [CrossRef]

42. Kelley, G.A.; Kelley, K.S.; Kohrt, W.M. Exercise and bone mineral density in men: A meta-analysis of randomized controlled trials. Bone 2013, 53, 103-111. [CrossRef]

43. Jensen, L.B.; Kollerup, G.; Quaade, F.; SØRensen, O.H. Bone Mineral Changes in Obese Women During a Moderate Weight Loss With and Without Calcium Supplementation. J. Bone Miner. Res. 2001, 16, 141-147. [CrossRef]

44. Villareal, D.T.; Fontana, L.; Weiss, E.P.; Racette, S.B.; Steger-May, K.; Schechtman, K.B.; Klein, S.; Holloszy, J.O. Bone Mineral Density Response to Caloric Restriction-Induced Weight Loss or Exercise-Induced Weight Loss: A Randomized Controlled Trial. Arch. Intern. Med. 2006, 166, 2502-2510. [CrossRef]

45. Radak, T.L. Caloric Restriction and Calcium's Effect on Bone Metabolism and Body Composition in Overweight and Obese Premenopausal Women. Nutr. Rev. 2004, 62, 468-481. [CrossRef]

46. Qiao, D.; Li, Y.; Liu, X.; Zhang, X.; Qian, X.; Zhang, H.; Zhang, G.; Wang, C. Association of obesity with bone mineral density and osteoporosis in adults: A systematic review and meta-analysis. Public Health 2020, 180, 22-28. [CrossRef]

47. Chen, Y.-Y.; Fang, W.-H.; Wang, C.-C.; Kao, T.-W.; Chang, Y.-W.; Wu, C.-J.; Zhou, Y.-C.; Sun, Y.-S.; Chen, W.-L. Body fat has stronger associations with bone mass density than body mass index in metabolically healthy obesity. PLoS ONE 2018, 13, e0206812. [CrossRef]

48. Jeon, Y.K.; Lee, J.G.; Kim, S.S.; Kim, B.H.; Kim, S.-J.; Kim, Y.K.; Kim, I.J. Association between bone mineral density and metabolic syndrome in pre- and postmenopausal women. Endocr. J. 2011, 58, 87-93. [CrossRef] 
49. Chain, A.; Crivelli, M.; Faerstein, E.; Bezerra, F.F. Association between fat mass and bone mineral density among Brazilian women differs by menopausal status: The Pró-Saúde Study. Nutrition 2017, 33, 14-19. [CrossRef]

50. Madeira, E.; Mafort, T.T.; Madeira, M.; Guedes, E.P.; Moreira, R.O.; de Mendonça, L.M.C.; Lima, I.C.B.; de Pinho, P.R.A.; Lopes, A.J.; Farias, M.L.F. Lean mass as a predictor of bone density and microarchitecture in adult obese individuals with metabolic syndrome. Bone 2014, 59, 89-92. [CrossRef]

51. Pasco, J.A.; Gould, H.; Brennan, S.L.; Nicholson, G.C.; Kotowicz, M.A. Musculoskeletal deterioration in men accompanies increases in body fat. Obesity 2014, 22, 863-867. [CrossRef]

52. Lin, H.-H.; Hsu, H.-Y.; Tsai, M.-C.; Hsu, L.-Y.; Chien, K.-L.; Yeh, T.-L. Association between type 2 diabetes and osteoporosis risk: A representative cohort study in Taiwan. PLoS ONE 2021, 16, e0254451. [CrossRef]

53. Asokan, A.G.; Jaganathan, J.; Philip, R.; Soman, R.R.; Sebastian, S.T.; Pullishery, F. Evaluation of bone mineral density among type 2 diabetes mellitus patients in South Karnataka. J. Nat. Sci. Biol. Med. 2017, 8, 94-98. [CrossRef] [PubMed]

54. Kameda, T.; Mano, H.; Yuasa, T.; Mori, Y.; Miyazawa, K.; Shiokawa, M.; Nakamaru, Y.; Hiroi, E.; Hiura, K.; Kameda, A.; et al Estrogen Inhibits Bone Resorption by Directly Inducing Apoptosis of the Bone-resorbing Osteoclasts. J. Exp. Med. 1997, 186, 489-495. [CrossRef] [PubMed]

55. Li, H.; Wallin, M.; Barregard, L.; Sallsten, G.; Lundh, T.; Ohlsson, C.; Mellström, D.; Andersson, E.M. Smoking-Induced Risk of Osteoporosis Is Partly Mediated by Cadmium from Tobacco Smoke: The MrOS Sweden Study. J. Bone Miner. Res. 2020, 35, 1424-1429. [CrossRef] [PubMed]

56. Weaver, C.M.; Alexander, D.D.; Boushey, C.J.; Dawson-Hughes, B.; Lappe, J.M.; LeBoff, M.S.; Liu, S.; Looker, A.C.; Wallace, T.C.; Wang, D.D. Calcium plus vitamin D supplementation and risk of fractures: An updated meta-analysis from the National Osteoporosis Foundation. Osteoporos. Int. 2016, 27, 367-376. [CrossRef] [PubMed]

57. Mahdi, A.A.; Brown, R.B.; Razzaque, M.S. Osteoporosis in Populations with High Calcium Intake: Does Phosphate Toxicity Explain the Paradox? Indian J. Clin. Biochem. 2015, 30, 365-367. [CrossRef]

58. Shih-Ying, C.; Jia-Rong, L.; Tzu-Hsiu, C.; Shiou-Guei, G.; Mei-Ding, K.; Wen-Harn, P. Dietary Supplements Usage Among Elderly Taiwanese During 2005-2008. Asia Pac. J. Clin. Nutr. 2011, 20, 327-336. [CrossRef]

59. Evans, R.G.; Stoddart, G.L. Consuming Research, Producing Policy? Am. J. Public Health 2003, 93, 371-379. [CrossRef] [PubMed]

60. Meyer, H.E.; Henriksen, C.; Falch, J.A.; Pedersen, J.I.; Tverdal, A. Risk factors for hip fracture in a high incidence area: A case-control study from Oslo, Norway. Osteoporos. Int. 1995, 5, 239-246. [CrossRef]

61. Bacon, W.E.; Hadden, W.C. Occurrence of Hip Fractures and Socioeconomic Position. J. Aging Health 2000, 12, 193-203. [CrossRef]

62. Kim, J.; Lee, J.; Shin, J.Y.; Park, B.J. Socioeconomic disparities in osteoporosis prevalence: Different results in the overall Korean adult population and single-person households. J. Prev. Med. Public Health Yebang Uihakhoe Chi 2015, 48, 84-93. [CrossRef]

63. Yu, W.-S.; Wang, C.-H.; Kuo, N.-W. Impact of Urbanization and Sunlight Exposure on Cataract Incidence. Appl. Sci. 2021, 11, 8137. [CrossRef] 